Methods Within the Idiopathic Urethritis Men's Project cohort study, we recruited men with NGU. NGU was diagnosed by the presence of urethritis signs and/or symptoms and urethral Gram stain with $\geq 5 \mathrm{PMN} / \mathrm{HPF}$. Men were treated with $1 \mathrm{gm}$ azithromycin and returned for a 1-month test-of-cure visit. At the test-of-cure visit, men were asked about post-treatment symptom outcomes and partner treatment. A first-catch urine specimen was obtained at both visits for five-pathogen testing for Neisseria gonorrhoeae (NG),Chlamydia trachomatis (CT), MG, Trichomonas vaginalis (TV), and Ureaplasma urealyticum (UU). NG-positive cases were excluded and five-pathogen-negative cases were classified as idiopathic urethritis (IU). Posttreatment symptom outcomes were: (1) resolved, (2) resolved then recurred, or (3) persisted unchanged.

Results One hundred twenty-four men are included in this study. The median age was 28, 52\% were African American, and $86 \%$ self-identified as heterosexual. All men reported urethral symptoms and $98 \%$ had a discharge on exam at baseline. Symptoms resolved completely in 91 (73\%) men. Symptoms resolved then recurred or persisted unchanged in $12(10 \%)$ and 21 (17\%) men, respectively. Excluding men with untreated partners $(\mathrm{N}=9,28 \%)$, a different pathogen was identified in $5(50 \%)$ and $4(25 \%)$ men with recurrent and persistent symptoms, respectively. In men with the same pathogen identified $(\mathrm{N}=15), 53 \%$ were $\mathrm{IU}, 33 \%$ were $\mathrm{MG}$, 7\% were CT, and 7\% were UU.

Conclusion Persistent NGU occurs in approximately 25\% of azithromycin-treated men and is related to a new infection in up to $50 \%$ of cases. In men with persistent symptoms and the same infection identified at the test-of-cure visit, MG and IU comprised $86 \%$ of cases, which suggests that MG and IUassociated organisms may be resistant to azithromycin.

Disclosure No significant relationships.

\section{P796 REASSESSING THE GRAM STAIN SMEAR (GSS) POLYMORPHONUCLEAR LEUKOCYTE (PMN) CUTOFF FOR DIAGNOSING NON-GONOCOCCAL URETHRITIS (NGU)}

${ }^{1}$ Gina Leipertz*, 'Laura Chambers, ${ }^{2}$ Sylvan Lowens, ${ }^{2}$ Jennifer Morgan, 'Sarah Romano, ${ }^{1}$ Tashina Robinson, ${ }^{3}$ Lindley Barbee, ${ }^{3}$ Matthew Golden, 'Lisa Manhart. 'University of Washington, Epidemiology, Seattle, USA; ${ }^{2}$ Public Health - Seattle and King County, Seattle, USA; ${ }^{3}$ University of Washington, Medicine, Seattle, USA

10.1136/sextrans-2019-sti.850

Background Recommended cutoffs for PMNs per high-power field (hpf) to define NGU vary. CDC treatment guidelines specify $\geq 2$ PMNs/hpf. Other guidelines recommend $\geq 5$ PMNs/hpf.

Methods From 08/2014-08/2018, we enrolled symptomatic and asymptomatic male STD clinic patients $\geq 16$ years with exclusively female partners in the past year. Men with gonorrhea or antibiotic use in the past month were excluded. We collected a urethral swab for GSS and urine for Chlamydia trachomatis(CT) and Mycoplasma genitalium (MG) testing (Aptima, Hologic). We calculated Youden's Index (J=sensitivity +specificity-1), which maximizes sensitivity and specificity, and calculated the proportions of CT/MG cases missed and cases treated in the absence of CT/MG (test-negative) for three PMN/hpf cutoffs. CT/MG co-infections $(\mathrm{N}=3)$ were excluded. Results Among 369 participants, median age was 32 (range 17-71), 53\% were white, and 25\% were black. Among all men with $0-1,2-4,5-9$, and $\geq 10 \mathrm{PMNs} / \mathrm{hpf}$, CT prevalence was $1 \%, 5 \%, 11 \%$, and $26 \%$, respectively; MG prevalence was $5 \%, 3 \%, 15 \%$, and $17 \%$. J was maximized at $\geq 5$ PMNs/ hpf for CT, MG, and CT/MG. Thirteen percent, 17\%, and $33 \%$ of $\mathrm{CT} / \mathrm{MG}$ cases were missed at the $\geq 2, \geq 5$, and $\geq 10$ PMNs/hpf cutoffs, respectively; 45\%, 33\%, and 21\% of testnegative cases were treated. Among symptomatic men ( $\mathrm{N}=166)$ with $0-1,2-4,5-9$, and $\geq 10 \mathrm{PMNs} / \mathrm{hpf}$, CT prevalence was $0 \%, 20 \%, 12 \%$, and $31 \%$, respectively; MG prevalence was 9\%, 0\%, 18\%, and 19\%. J was maximized at $\geq 5$ PMNs/hpf for MG, and $\geq 10 \mathrm{PMNs} / \mathrm{hpf}$ for CT and CT/MG. Five percent, $8 \%$, and $25 \%$ of $\mathrm{CT} / \mathrm{MG}$ cases were missed at the $\geq 2, \geq 5$, and $\geq 10 \mathrm{PMNs} /$ hpf cutoffs, respectively; $72 \%$, $64 \%$, and $43 \%$ of test-negative cases were treated.

Conclusion The increase in missed CT/MG cases between the $\geq 2$ PMNs/hpf cutoff and $\geq 5$ PMNs/hpf cutoff was minimal; the $\geq 5 \mathrm{PMNs} / \mathrm{hpf}$ cutoff treats fewer cases without CT/MG. The $\geq 5 \mathrm{PMNs} / \mathrm{hpf}$ cutoff appears optimal in this population.

Disclosure No significant relationships.

\section{P797 ANTIBODY RESPONSE TO MYCOPLASMA GENITALIUM IN LONGITUDINALLY INFECTED MEN WITH NON- GONOCOCCAL URETHRITIS}

${ }^{1}$ Gwendolyn Wood*, ${ }^{1}$ Stefanie Iverson Cabral, ${ }^{2}$ Lisa Manhart, ${ }^{3}$ Sylvan Lowens, ${ }^{2}$ Catherine Gillespie, ${ }^{4}$ Patricia Totten. 'University of Washington, Seattle, Seattle, USA; ${ }^{2}$ University of Washington, Epidemiology, Seattle, USA; ${ }^{3}$ Public Health - Seattle and King County, Seattle, USA; ${ }^{4}$ University of Washington, Infectious Diseases, Seattle, USA

\subsection{6/sextrans-2019-sti.851}

Background A sensitive and specific serologic test is needed to evaluate the association of Mycoplasma genitalium (MG) infection with serious upper reproductive tract sequelae in women. In this study, we compared the ability of immunoblot and ELISA methods to detect serum antibody reactivity with the immunodominant $\mathrm{MgpB}$ and $\mathrm{MgpC}$ adherence proteins among MG-infected men with nongonococcal urethritis (NGU).

Methods Serum samples collected at two time points (spanning 15-86 days) from 22 MG-infected, PCR-positive men with NGU were assayed for reactivity to MG whole cell lysates by immunoblot, and to the conserved C-terminus of $\mathrm{MgpB}$ by ELISA, compared to 19 MG-negative controls. Additionally, we selected six $\mathrm{MG}(+)$ men with a variety of immunoblot reactivities and examined their serum specimens for ELISA reactivity to 16 recombinant peptides spanning conserved and variable domains of $\mathrm{MgpB}$ and $\mathrm{MgpC}$ at two time points.

Results Among men with current MG infection, immunoblot detection of $\mathrm{MgpB}$ antibodies outperformed an ELISA assay detecting reactivity to the conserved C-terminus of $\mathrm{MgpB}$ with $90.9 \%$ and $81.8 \%$ sensitivity, and $92.3 \%$ and $82.3 \%$ specificity, respectively. In contrast to immunoblot results, ELISAreactivity to individual peptides spanning $\mathrm{MgpB}$ and $\mathrm{MgpC}$ indicated patient antibodies more frequently targeted the Cterminus of $\mathrm{MgpC}$ than the C-terminus of MgpB. As expected, most patient sera reacted poorly in ELISAs to recombinant peptides spanning the $\mathrm{MgpB}$ and $\mathrm{MgpC}$ variable regions as these sequences corresponded to the G37 type strain rather than the infecting strains.

Conclusion Our findings suggest that an MG ELISA test could be improved by including conserved portions of both the $\mathrm{MgpB}$ and $\mathrm{MgpC}$ proteins, providing an alternative to the more labor intensive immunoblot. Such a test will be especially valuable for associating current or past MG infection with serious upper reproductive tract disease in women.

Disclosure No significant relationships. 